\title{
Bartolomeo Borghesi, Theodor Mommsen et l'édition des inscriptions de Pirro Ligorio dans le Corpus Inscriptionum Latinarum (cIL)
}

\author{
Ginette Vagenheim
}

Au moment d'envoyer à l'Académie des Sciences de Berlin son plan pour l'édition d'un Corpus des inscriptions latines (CIL) en 1847, Théodor Mommsen (1817-1903) a conscience du problème que posent les fausses inscriptions et notamment celles attribuées à Pirro Ligorio (1512-1583), considéré comme le plus grand faussaire en épigraphie de tous les temps. Le présent article explore pour la première fois la genèse de l'édition des 'falsae ligorianae' dans le cIL en rappelant d'abord l'existence, trop souvent oubliée, d'un premier projet de CIL confié à Olaus Christian Kellermann sous la direction officieuse de Bartolomeo Borghesi (1781-1860); à travers leur correspondance s'élaborent les principes méthodologiques qui seront mis en œuvre dans le second projet du clL après que Mommsen ait pris la décision de créer une catégorie d'inscriptions consacrée aux 'falsae vel dubiae'; le savant allemand mettait ainsi à la disposition des générations futures un matériel encore trop souvent écarté de la recherche historique.

When Theodor Mommsen sent to the Academy of Sciences in Berlin his plan for the publication of a corpus of Latin inscriptions (CIL) in 1847, he was aware of the problems that had arisen due to forgeries, and especially those attributed to Ligorio, who was considered the greatest forger of all time in epigraphy. This article explores for the first time the genesis of the publication of the 'falsae ligorianae' in CIL, recalling first the existence, often forgotten, of a first project of a clL entrusted to Olaus Christian Kellermann under the unofficial direction of Borghesi; through their correspondence they formulated the methodological principles which would be followed in the second project of a CIL after Mommsen had taken the decision to create a specific category of inscriptions 'falsae vel dubiae'; the German scholar thus provided for future generations a material that remains little explored in the field of epigraphy.

Fino dai primi tempi, il famigerato Pirro Ligorio non aveva risparmiato di contaminare anche la propria patria colle sue imposture.

Compte rendu de Borghesi de l'édition de Mommsen des Inscriptiones Regni Neapolitani Latinae (I852).

QuAND Théodor Mommsen envoie à l'Académie des Sciences de Berlin son plan pour l'édition d'un corpus des inscriptions latines (Über Plan und Ausführung eines Corpus Inscriptionum Latinarum, janvier I847), il a conscience, du moins en théorie, du problème que pose la falsification et en particulier les fausses inscriptions attribuées à Ligorio. C'est pour cette raison qu'il inclut dans son plan un chapitre consacré à la Kritik der Achteit dans lequel il propose d'examiner en priorité les Fälschungen des Ligorius conservées dans sa grande encyclopédie de la civilisation gréco-romaine en quarante-deux volumes (Antichità romane); ${ }^{1}$ ce chapitre trouve place à son tour dans la troisième partie du projet, dédiée à la Kritik der Inschriften: avant cela, Mommsen avait divisé l'étude des inscriptions en deux parties, la première consacrée aux inscriptions conservées (die Steine) et la deuxième aux inscriptions transmises uniquement à travers les éditions et les manuscrits (die Literatur); concernant ce deuxième groupe d'inscriptions, le savant allemand souligne d'emblée l'exigence de le soumettre à un examen philologique dans le but d'en établir l'authenticité (Kritik der Achteit). ${ }^{2}$

Quelle méthode Mommsen allait-il mettre en place pour distinguer les vraies inscriptions des fausses? Pour le découvrir, il faut revenir au premier projet de constitution d'un Corpus Inscriptionum Latinarum (CIL), méconnu aujourd'hui mais qui 
pourtant coïncide avec une étape fondamentale de l'histoire de l'épigraphie au XIX ${ }^{\mathrm{e}}$ siècle: celle des rapports entre Borghesi et les savants étrangers, germanophones puis français, venus à Rome en tant que diplomates, comme Barthold George Niebuhr (I776-I83I) et Christian Bunsen (I79I-I860) - ou en tant que savants, notamment après la création de l'Instituto di corrispondenza archeologica (I 829). Borghesi en fut dès la création un membre actif, secrétaire de la section italienne; c'est dans ce cadre qu'il rencontre le jeune savant danois Olaus Christian Kellermann (I805-37) dont il soutient ardemment le projet d'un recueil d'inscriptions latines; à travers leur correspondance se dessinent peu à peu les grands principes méthodologiques qui allaient présider à l'entreprise du $C I L$; ils seront à nouveau évoqués, quelques années après l'arrêt brutal du projet dû à la mort de Kellermann (I837), dans les lettres entre Borghesi et les autres membres de l'Institut, en particulier ceux qui s'intéressent à l'épigraphie, tels qu'August Emil Braun (I809-56), Eduard Gerhard ( I 795-I867), Wilhelm Henzen ( I 8 г6-87), Otto Jahn ( 18 I 3-69) et Mommsen. ${ }^{3}$

Dix ans après la disparition de Kellermann, Mommsen reprendra le flambeau et mettra en œuvre les principes énoncés par Borghesi, d'abord en choisissant le classement topographique des inscriptions, brillamment illustré dans son édition des Inscriptiones Regni Neapolitani Latinae (I862), mais surtout en affrontant dès le début l'épineuse question des inscriptions ligoriennes que le jeune Danois n'avait pas eu le temps d'aborder; Mommsen finira par y apporter une solution d'ordre méthodologique dont nous suivrons l'élaboration au fil de sa correspondance avec Borghesi.

\section{Le premier projet d'un CIL}

Kellermann arrive à Rome en $\mathrm{I} 83 \mathrm{I}$ à la faveur d'une bourse du gouvernement danois ${ }^{4}$ et dès 1833 , il entretient une correspondance suivie avec Borghesi, notamment autour de son édition des Vigilum Romanorum latercula duo Coelimontana magnam partem militiae Romanae explicantia ${ }^{5}$ celle-ci paraît en I835 au retour d'un séjour du jeune chercheur à San Marino, qui inaugure une longue tradition d'ascension du mont Titan par les savants venus de toute l'Europe pour profiter de l'enseignement de Borghesi. ${ }^{6}$ Cette première publication épigraphique avait pour but de montrer les capacités du jeune homme à mener à bien la publication d'un corpus d'inscriptions latines.

Quelques jours après la visite de Kellermann, Borghesi réaffirme, par écrit cette fois, sa conception du futur recueil épigraphique: il aura la forme d'un supplément aux grands corpus d'inscriptions, depuis celui de Janus Gruterus ( ( 560-I627) jusqu'à l'édition d'Orelli ( $1787-$ I 849): ${ }^{7}$

Intendo dire la collezione delle iscrizioni latine, o nuove, o stampate parzialemente, lasciando da parte le greche, perché a queste si è già previsto dal Boeckh. Io vorrei ch'estendesse le sue conquiste a tutto ciò che non trovasi nel Grutero, nel Reinesio, nello Spon, nel Doni, nel Gudio, nel Fabretti, nel Maffei, nel Muratori, nel Donati, negli Arvali del Marini e nell'Orellio ${ }^{8}$ saccheggiando e spogliando poi impunemente qualunque altra raccolta o museo ... Non le dissimulo che l'impresa è gigantesca, e credo di essere moderato nel calcolare almeno a ventimila le iscrizioni che si radunerebbero. $\mathrm{Vi}$ ha in ciò di che sgomentare chiunque, e da far temere che non basti la vita per tanto lavoro. Niuno più di me può sentire questo pericolo per la voglia che mi prese troppo giovanilmente di radunare non solo i nomi di tutti i consoli, ma tutte le notizie che si hanno di loro (3I janvier I835).

Au cours de cette même année i 835, juste avant de quitter Rome pour quelques temps, Kellermann crée la surprise en annonçant à Borghesi qu'il a l'intention de publier un nouveau corpus d'inscriptions latines. Borghesi félicite le jeune homme pour cette entreprise qui fera pendant au corpus des inscriptions grecques d'August Boeckh ${ }^{9}$ et à laquelle il prédit le même accueil de la part de la communauté internationale ${ }^{10}$ il offre également à Kellermann sa coopération totale ainsi que la mise à disposition de ses travaux épigraphiques $;{ }^{11}$ puis, revenant au projet du corpus, Borghesi rappelle que le plus grand bénéfice de la nouvelle collection épigraphique ne réside pas tant dans la publication de textes nouveaux que dans la correction qu'il apportera aux inscriptions, déjà connues et qui aboutira à éliminer les fausses inscriptions principalement celles de Ligorio. Or, on ne pourra s'en débarrasser tant que ses manuscrits resteront inédits:

Ma non è tanto per l'accrescimento di nuove cognizioni, che io La felicito della sua idea, quanto per la rettificazione delle antiche. Sarebbe certamente un gran merito quello di far sparire un'infinità di false lezioni, e di decidere così una quantità di controversie che hanno diviso gli antiquari. Ma il vantaggio principale per me, vantaggio che non può ottenersi se non coll'impresa da Lei immaginata, sarebbe quello di togliere una volta allo studio dei dotti le imposture del Ligorio, moltissime delle quali restano mascherate ancora, perché i suoi sessanta e più libri non sono mai stati stampati giammai. 
Borghesi avait eu affaire à Ligorio dans le contexte de la découverte de deux nouveaux grands fragments des Fastes consulaires qui avait eu lieu au Forum romain à l'automne i 8 I 6 ; Borghesi s'était alors précipité à Rome, comme l'avaient fait trois cents ans avant lui, tous les érudits présents en Italie à l'annonce de la découverte des premiers fragments en I $546 .{ }^{12}$ L'archéologue Carlo Fea $\left(\mathrm{I}_{753}-\mathrm{I} 836\right)$, qui avait découvert les fragments, eu cependant l'intelligence d'en confier l'étude à Borghesi qui en assura la publication (Nuovi frammenti dei fasti consolari capitolini) $;{ }^{13}$ au cours de son édition, Borghesi avait consulté toutes les publications antérieures des Fastes mais aussi les recueils manuscrits des humanistes qui les avaient édités au $\mathrm{XVI}^{\mathrm{e}}$ siècle; c'est ainsi qu'il découvrit que tous ces manuscrits étaient remplis de fausses inscriptions ligoriennes; celles-ci étaient ensuite passées dans les grands recueils imprimés, sans interruption jusqu'à Orelli; mais avant de s'en rendre compte, Borghesi avait perdu des années à tenter de recomposer le texte des Fastes pollué par plus de 400 inscriptions fausses:

Quando Ellà sarà entrata ben a dentro al suo lavoro, si stupirà della quantità degli aborti di costui, che sotto gli onesti nomi dell'Orsino, del Panvinio, del Manuzio, del Gutenstenio, del Vallamberto, del Langermanno, e di tanti altri, si sono insinuati nel Gruter, nel Reinesio, nel Muratori, nel Fabretti, nello Spon, come toccherà con mano studiando i manoscritti di quel falsario nella Vaticana. Anche l'Orelli, malgrado la sua critica diligente, vi è rimasto gabbato più volte. Io posso dirne qualcosa, perché ho perduto inutilmente degli anni nel combinare nei fasti un mucchio d'iscrizioni ricalcitranti, che in ultimo ho poi trovato provenire da quell'impostore, per cui più di quattrocento ho dovuto relegare fra le apocrife.

Borghesi était l'un des rares savants à avoir vu les manuscrits de Ligorio, sauf ceux qui se trouvaient à Naples, difficilement accessibles alors comme de nombreuses collections; il en était contrarié parce qu'il ne pouvait vérifier si certaines inscriptions qui lui semblaient suspectes venaient effectivement de Ligorio; et c'est pourquoi Borghesi rappelle une fois de plus à Kellermann la nécessité de consulter les manuscrits pour éviter les erreurs de ses prédécesseurs, y compris Orelli; ce dernier était même tombé dans l'erreur inverse et avait exclu de son corpus des inscriptions qui existaient réellement et qui étaient donc authentiques:

Né burlo quando le dico che le nostre grandi collezioni, meno quelle del Maffei e del Marini, sono piene di lapidi false . . . [che] si sono insinuati nel Grutero, nel Reinesio nel Muratori, nel Fabretti, nello Spon. Pochi hanno potuto accorgersene perché pochi hanno veduto la copia dei manoscritti ligoriani nella Vaticana ed io sono forse il solo cui sia stato concesso esaminare con agio gli originali conservati con gran segreto negli archivi di corte a Torino . . Mi duole che per la circostanza dei tempi non mi riuscisse altrettanto quando fui a Napoli riguardo agli pochi volumi di quell'impostore che ivi sono pervenuti dall'eredità Farnese e che per ciò mi restino tuttavia delle iscrizioni nelle quali sento l'odore ligoriano ma da cui non posso certificare l'origine. L'Orelli ha fatto indubitamente del gran bene ma per mancanza di queste cognizioni precise è avvenuto che anch'egli sia rimasto allucinato talvolta, ricevendo senza difficoltà delle lapidi che provengono dal Ligorio, mentre invece ne ha rigettate delle altre ch'esistono realmente e che sono sincerissime.

Kellermann avait bien entendu ces conseils de Borghesi et c'est ainsi qu'il propose, dès le premier paragraphe du Denkschrift qu'il envoie à l'Académie de Berlin ( I juin I836), l'examen systématique des éditions et des manuscrits épigraphiques, en des termes qui font écho à ceux de son maître: ${ }^{14}$

Von 50 oder 60,000 lateinischen Inschriften welche diese Sammlungen enthalten mögen, sind sehr viele und wol bei weitem mehr als insgemein angenommen wird, falsch, und verdanken grösstenteils dem Pirrus Ligorius unseligen Andenkens ihre existenz. Die anscheinende Reichhaltigkeit und Wichtigkeit der mehr als von Ligorius handschriftlich hinterlassenen Bücher imponierte dem Ursinus, Panvinius, Manutius, Gutenstenius, Wallambert, Langermann und vielen andern höchst verdienten Gelehrten dergestalt dass sie ihre achtbaren und keinen verdacht erregenden Namen dazu hergaben fast alle jene falschen produkten in die grossen sammlungen einzuschwärzen, so dass wol nur Maffei und Marini ganzfrei dasthehen. ${ }^{15}$

Dans une lettre de la même année, Borghesi énonce un second principe à appliquer à l'étude des inscriptions ligoriennes, qui consiste à rejeter tout texte épigraphique - manuscrit ou imprimé - qui ne serait connu que par Ligorio, et inversement à considérer comme authentique toute inscription du faussaire qui serait transmise également par ses contemporains dignes de foi, tels que l'érudit flamand Martinus Smetius (I 525-78), le bourguignon Jean Matal (I 5 I 797), l'helléniste Benedetto Egio de Spolète ( $\uparrow$ après I 57 I), l'érudit de Sienne Celso Cittadini ( $5_{553}$-I627) ou le Romain Aldo Manuzio junior ( $1547-97$ ):

... il canone generale che tutti i marmi del Ligorio, che non sono stati veduti da alcuno dei suoi contemporanei in Roma, come lo Smezio, il Metello, l'Egio, il Cittadini e simili si hanno da credere ragionevolmente finti da lui ...

Il ne restait plus à Kellermann que de se mettre au travail d'autant plus que Fea, fervent défenseur de 
Ligorio, venait de mourir, ce qui levait, aux dires de Borghesi, le dernier obstacle à la mise en œuvre d'une véritable étude critique des inscriptions ligoriennes:

A quest'ultimo avanzo della grande scuola degli antiquari romani del secolo passato (i.e. Fea), quantunque ne fosse indubitatamente il più debole, aveva però grande influenza nel governo, né poteva al certo applaudire in suo cuore ad un lavoro diretto precipuamente a bandire le falsità del Ligorio di cui si è mostrato sempre caldo fautore.

L'archéologue, qui avait dirigé les fouilles au Forum en I 8I6, avait pu constater sur le terrain la qualité des copies d'inscriptions de Ligorio ainsi que l'exactitude de ses notices archéologiques:

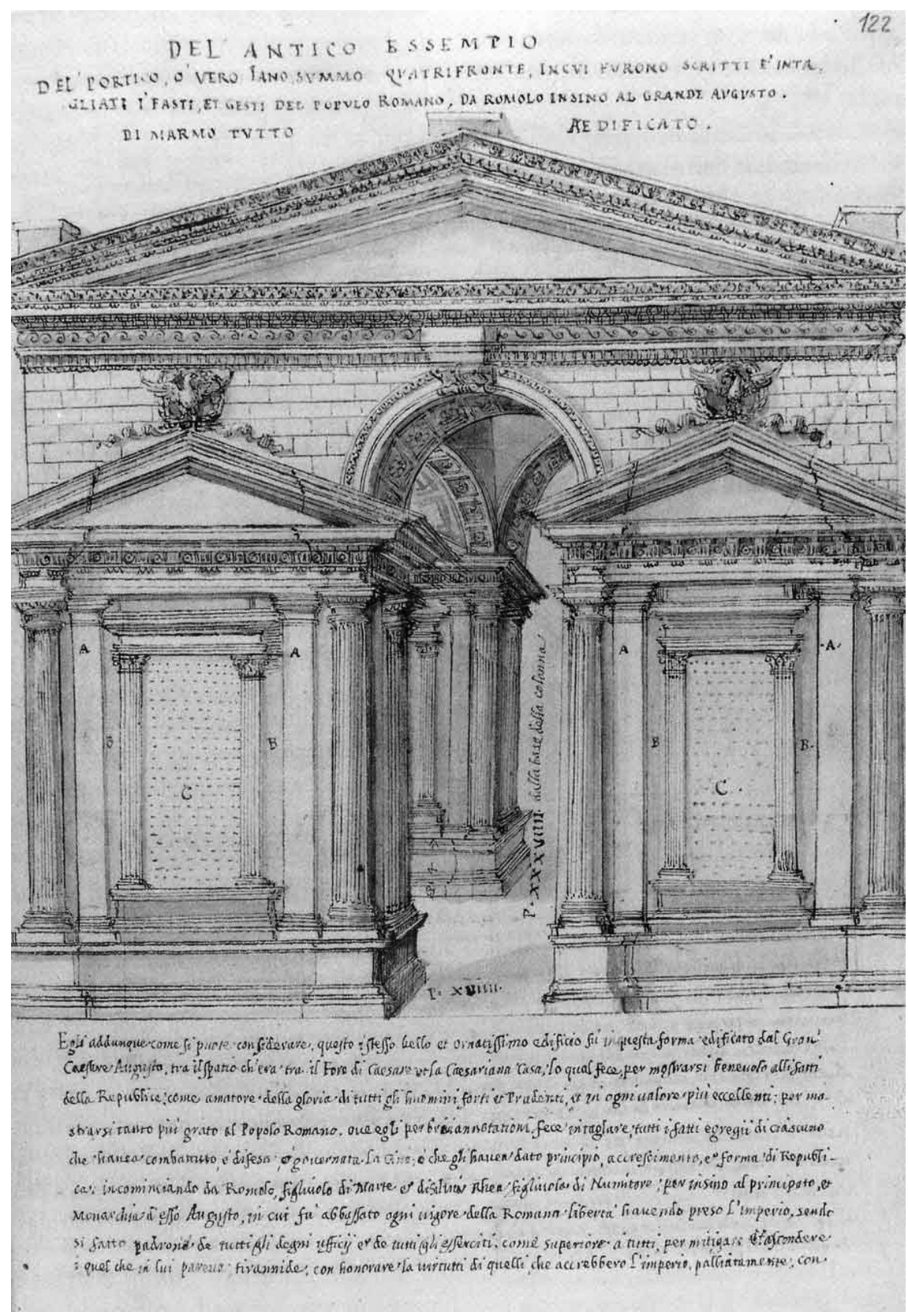

Fig. I. Pirro Ligorio, Arc à quatre faces. Turin, Archivio di Stato, vol. 5 fol. I I 2 r. 
Io ho avuto occasione di vedere gli originali di tante lapidi da lui riportate e le ho trovate più o meno ben riferite . . . Sopratutto il Ligorio è interessante per le notizie degli scavi che dà; e della località che sempre sono state omesse da chi ha dato copie delle di lui iscrizioni. ${ }^{16}$

Dans le cas des Fastes capitolins, Fea avait eu raison de soutenir Ligorio qui affirmait que le monument sur lequel avaient été gravés les textes à l'époque d'Auguste n'était pas un hémicycle mais un arc à quatre faces dont il fit deux dessins (Figs I-2); ${ }^{17}$ Ligorio allait ainsi à l'encontre des érudits chargés d'éditer le texte des fastes, notamment Onofrio Panvinio ( $1530-$ I 568), Antonio Agustín ( I 5 I 7-86) et Ottavio Pantagato (I494-I567) que Borghesi considérait comme des témoins dignes de foi. Un an après Fea, Kellermann disparaît à son tour, succombant à une épidémie de choléra à Rome; sa mort priva d'un seul coup Borghesi d'un ami et de son projet d'un CIL.

\section{Le second projet de $\mathrm{ClL}$}

Dans une lettre du 29 mars I 844 à son ami Braun, Borghesi expose la méthode que devra suivre le

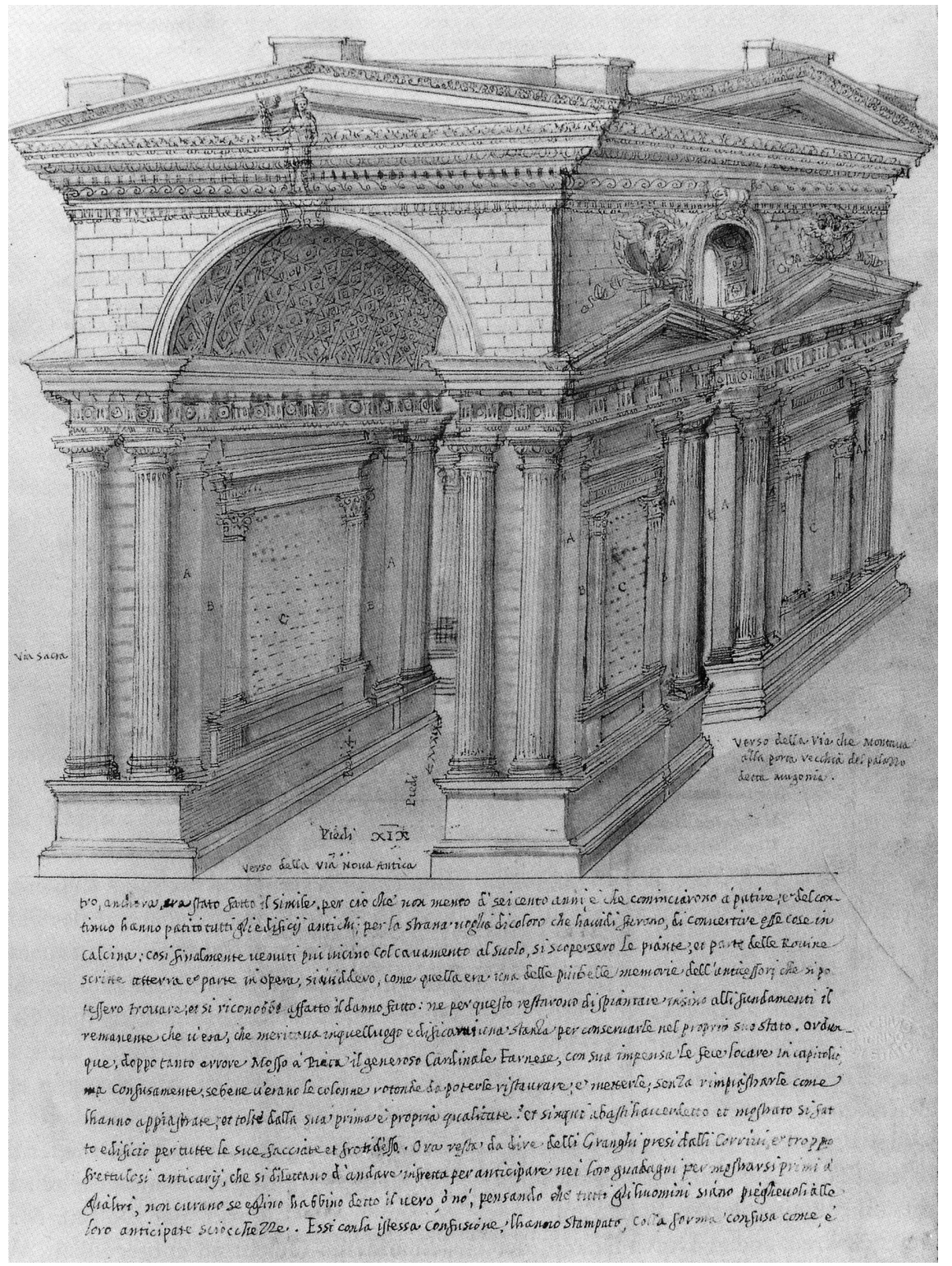

Fig. 2. Pirro Ligorio, Arc à quatre faces. Turin, Archivio di Stato, vol. I 5, fol. I23r. 
successeur de Kellermann pour l'édition du $C I L .{ }^{18}$ Quand paraît le corpus des inscriptions latines du Règne de Naples (IRNL, I862), Mommsen expose la méthode utilisée dans l'examen des manuscrits ligoriens, dans la célèbre lettre de dédicace à son magister, patronus amicus:

Summa res eo vertitur, quod non tam inscriptiones singulas in iudicium vocavi, quam singulos auctores . . . legem secutus quae in foro obtinet: dolum non praesumi sed probato dolo totum testem infirmari.

Le vœu de Borghesi est exaucé: Mommsen a débarrassé l'épigraphie napolitaine des fausses inscriptions de Ligorio:

Ma il principale merito di uno così utile lavoro si è quello di aver purgato finalmente l'epigrafia napoletana dalle molte lapidi fittizie o corrotte. Fino dai primi tempi il famigerato Pirro Ligorio non aveva risparmiato di contaminare anche la propria patria colle sue imposture. ${ }^{19}$

Si l'on poursuit la lecture de la dédicace, on découvre pourtant chez Mommsen une position moins radicale vis-à-vis du faussaire napolitain dont il a pu constater parfois la bonne foi; si bien qu'il s'est parfois décidé à publier comme genuinae des inscriptions transmises seulement par Ligorio, tout en restant prudent:

Item si qui reperiuntur apud falsarium tituli, qui plane recedunt ab indole eius certa et nota, ut solemus Pratillianos et Ligorianos longo horum usu edocti tum quoque deprehendere, cum sub alio nomine eorum nugae circumferuntur, ita e contrario hos genuinis inserere ausus sum, numquam tamen nisi monito ut sibi caveat lectore.

L'analyse systématique des inscriptions ligoriennes ne pouvait toutefois être envisagée à ce stade de l'édition du Corpus où seul le principe radical énoncé par Borghesi permettait de mener le projet à terme; et c'est précisément au moment où il renonce à cette méthode intermédiaire que Mommsen a une intuition géniale: celle de publier toutes les inscriptions fausses ou douteuses dans une catégorie consacrée aux falsae vel suspectae - une catégorie qui n'avait pas été prévue dans le plan initial, et dont Borghesi, dans son compte rendu élogieux des IRNL, signalera l'existence et la place en fin de volume, après les index.

Borghesi n'attendra pas longtemps pour mesurer l'immense profit de cette importante décision méthodologique: dès qu'il vit, dans le chapitre des falsae vel suspectae, l'inscription $\mathrm{n}^{\circ} 209^{*}$ de Canosa, que Mommsen avait trouvée dans la vieille édition de Lupoli de I793 et qu'il avait écartée en raison d'aberrations dans l'onomastique de l'empereur Constantin, Borghesi lui fit parvenir la copie correcte de la même inscription qu'il avait découverte dans un manuscrit de Matal (Vat.lat.6o39, f.364), considéré comme un auteur digne de foi.

\section{$\mathrm{n}^{\circ}$ 209* Venusa ut aiunt Canusium translata Lup.}

Lupuli qui ait se exscripsisse. Constantinus saepissime maximus dicitur, nuтquam magnus; praeterea insolens est omissio nominum, magis etiam vivo Constantino patre filiis solis honor habitus. Vivo enim Constantino titulus positus sit necesse est, cum eo mortuo statim tres filii Augusti procederent.

\author{
SALVIS DDD NNN \\ CONSTANTINO AVG. ET \\ FILIIS EIVS. CAESS \\ VOLVSIVS VENVSTVS \\ VC. CORRECTOR \\ APVL [T CALAB $[\mathrm{sic}]$ \\ OB AMOREM \\ PATRIAE STATVAS \\ ORNATVI PORTICVS \\ POSVIT
}

Dès réception de la copie de Borghesi, Mommsen se hâta de réintroduire le texte de Canosa dans la section des genuinae cette fois, sous le $\mathrm{n}^{\circ} 7205:^{.0}$

\section{Canusii Metell.}

Canusium Venusia translatam fingit Lup.

Genuinum Metelli typum ex cod. Vat. 6039 p. 364 misit Borghesius; Lupuli it; Venus. p. 3 33,364 protulit interpolatam quem repetivi n. 209* additis rationibus propter quas inscriptio inter suspectas ableganda fuit. Iam sublatis naevis et a iusto vindice defensa iterum hic collocatur.

Le scénario de 'sauvetage' de l'inscription de Canosa, qui illustrait l'exemple à suivre dans l'examen des sources que Mommsen avait appelées Die Literatur, sera encore évoqué dans la lettre de dédicace à Borghesi confirmant ainsi sa portée méthodologique:

Legebaturque [in suspectarum ordine] re vera titulus Canusinus $n^{\circ} 7205$ (IX ${ }^{\circ} 329$ ), cui iam Tuo beneficio et accessit iusta Metelli auctoritas et detersae sunt sordes interpolationis Lupolianae. ${ }^{21}$

En effet, elle permit à Mommsen de prendre véritablement conscience de la nécessité de consulter les manuscrits et de ne pas se contenter de l'examen des imprimés, comme il l'avait fait, de manière générale pour la publication des IRNL; c'est aussi pour cette raison qu'il aura la grande idée d'associer 
à la direction du CIL celui qui mieux que quiconque connaissait les manuscrits: Giovanni Battista de Rossi (г 822-94), ${ }^{22}$ ses fonctions de scriptor Latinus à la Bibliothèque apostolique vaticane allait en outre permettre aux éditeurs du CIL d'accéder plus facilement aux nombreux recueils épigraphiques de ce fonds mais aussi des autres bibliothèques romaines. De Rossi possédait de rares compétences de paléographe, qu'il avait développées au cours de son étude des inscriptions chrétiennes et qui allaient se révéler précieuses dans l'identification des auteurs de recueils épigraphiques. Le savant romain fut le premier à réfuter l'identification de la main de Ligorio dans le célèbre Codex Ursinianus (Vat.lat.3439), identification dont Borghesi était l'auteur, mais il ne réussit pas à distinguer la main de Panvinio dans la plupart des copies de dessins ligoriens, ni celle de Stephanus Pighius. ${ }^{23}$ De même, si l'on doit à de Rossi d'avoir reconnu la main de Panvinio dans les deux recueils épigraphiques Vat. lat. 6035 et 6036 que Borghesi attribuait à Manuzio, dans ce cas non plus de Rossi ne remarqua pas la présence d'autres mains, dont celle de Ligorio ${ }^{24}$ et c'est ainsi que Mommsen publiera comme genuina, une inscription du Vat.lat.6o36 copiée par Ligorio. Il ne fait pas de doute qu'il l'aurait classée parmi les falsae s'il y avait reconnu la main de l'antiquaire; finalement l'inscription se révélera être bel et bien authentique. ${ }^{25}$

Le principe général énoncé par Borghesi, puis repris par Mommsen, fut suivi par tous les éditeurs des inscriptions ligoriennes, y compris ceux des inscriptions grecques; mais dans ce cas non plus, l'examen spécifique des inscriptions fausses, ne fut jamais négligé, comme l'illustre à plusieurs reprises Luigi Moretti, qui réhabilita, entre autres, l'inscription $I G$ XIV $3{ }^{\text {I }} 8^{* 26}$ (Fig. 3), conservée 'Nel ghetto di Roma, in una casa ch'è in piazza Giudea'. Georg Kaibel (I849-I90I) l'avait à tort classée parmi les falsae parce qu'elle venait de Ligorio:

Tamen cum Ligorio fides temporibus illis omnino improbaretur, nulla conquisitione vel collatione dignum Kaibel titulum putavit. Titulus qui hodie genuinus vindicatur, ostendit quam temere tituli permulti tamquam ficti damnati sint nulla alia ratione ab uno Ligorio traditi (IGUR $690) .^{27}$

On a vu que Borghesi avait critiqué l'attitude d'Orelli parce que ce dernier avait rejeté des inscriptions ligoriennes alors qu'elles 'existaient vraiment' ('ch'esistono veramente e che sono sincerissime'). Le principe qui sous-tend cette réflexion sera suivi à la lettre par Mommsen qui publiera parmi les genuinae les fausses inscriptions gravées sur pierre du Musée de Naples. ${ }^{28}$ C'est finalement son ami Henzen qui le premier attirera l'attention sur le phénomène de falsification sur pierre dans le cas des inscriptions ligoriennes. ${ }^{29}$

Si les fondateurs de l'épigraphie moderne se sont parfois fourvoyés, en écartant à tort une inscription ligorienne authentique ou en incluant dans leur corpus ses falsifications, il n'en reste pas moins qu'en nous procurant l'édition du $C I L$, ils nous ont permis l'accès à ces documents qui, bien au-delà du problème qu'ils posent sur le plan historique, témoignent d'une approche particulière de l'Antiquité qui mérite d'être étudiée dans son contexte, ${ }^{30}$ c'est-à-dire

\section{8* Nel muro d' una casa, ch' è in piazza Iudea.}

$$
\begin{aligned}
& \Gamma N \cdot K \wedge \cdot \Pi \wedge \omega K|\omega||\Pi A C| K P A T \in|\cdot \theta P \in| \Pi T \omega . \\
& |\triangle| \omega \cdot M N \cdot X A P \mid N \cdot \in C \Theta \wedge H \cdot \Theta
\end{aligned}
$$

Ligorius Neap. VII 476 (Ursin. Vat. 3439 f. 124, Suares. Vat. 9139

f. 85); ex Ligorio Murat. 1451, 8; hinc Franz 6609. - Verba «นท́|«ฑs

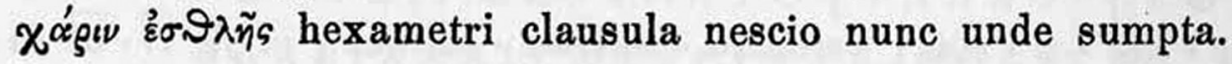

Fig. 3. Ig XIV 3I 8*. Pirro Ligorio, Naples, Biblioteca nazionale, XIII.B.7, p. 476. 
celui de la réception de la culture classique à la Renaissance.

\section{Adresse pour correspondance}

Ginette Vagenheim, Université de Rouen, UFR de Lettres et Sciences Humaines, F-761 23 Mont-St-Aignan Cédex.

ginette.vagenheim@univ-rouen.fr

\section{Notes et references}

I Je remercie Marco Buonocore, direttore della sezione archivi et scriptor latinus à la Bibliothèque apostolique vaticane, pour sa lecture attentive et ses précieuses corrections, notamment dans la transcription des textes: toutes les erreurs qui subsistent me sont entièrement imputables. Les volumes sont dispersés entre Naples (Biblioteca nazionale, XIII.B.I-Io), Turin (Archivio di Stato, Taur. I-30), Oxford (Bodleian Library, Canon.ital. I38) et Paris (Bibliothèque nationale de France, Paris. Ital. I 129 ).

2 L. Wickert, Theodor Mommsen. Eine Biographie, tomes I-III (Frankfurt am Main, I962-9).

3 Sa correspondance est publiée dans les tomes VI-VIII des CEuvres complètes, à Paris, par l'imprimerie impériale, en I 868 (VI) et I 872 (VII-VIII), avec un index des correspondants et un index analytique dans le tome Ix (I893).

4 Sur Kellermann, O. Jahn, Specimen epigraphicum in memoriam Olai Kellermann (Kiel, I84I).

5 Ce dernier avait interdit à Kellermann de faire allusion à cette collaboration: 'Ella vedrà abbastanza la ridicolezza cui andremmo incontro ambedue, se dirigendomi la sua operetta volesse in qualche caso citarmi. Intendo che i fogli che Le invio lo siano in tutta segretezza. Spessissimo ci saremo incontrati nelle stesse opinioni e ciò sarà una prova che non ci siamo ingannati. Altra volta i nostri pareri non saranno concordi, ed Ella, avendole manifestato il mio, potrà pesarne il valore, libera sempre di accettarlo o di rigettarlo.' Oeuvres, op. cit. (note 3), tome VII, p. 40.

6 La liste des savants qui gravirent le Mont Titan se trouve dans A. Campana, 'Bartolomeo Borghesi (Bartolino)', Dizionario biografico degli italiani, vol. I2 (I97I), dont je me suis beaucoup servie pour cet article.

7 J. Gruterus, Inscriptiones antiquae totius orbis romani, in absolutissimum corpus redactae (Heidelberg, I603); J. C. Orellius, Inscriptionum Latinarum selectarum amplissima collectio (Zurich, I828).

8 Th. Reinesius, Syntagma inscriptionum antiquarum (Leipzig, I682); J. Sponius, Miscellanea eruditae antiquitatis (Lyon, I685); J. B. Donius, Inscriptiones antiquae (Florence, I73I); M. Gudius, Antiquae inscriptiones quum graecae tum latinae (Leeuwarden, I73I); R. Fabrettius, Inscriptionum antiquarum explicatio (Rome, I702); S. Maffeius, Museum veronense (Verona, I799); L. A. Muratori, Novus thesaurus veterum inscriptionum (Milan, I739); S. Donati, Ad novum thesaurum veterum inscriptionum (Lucca, I765); G. Marini, Gli atti e monumenti de' fratelli Arvali (Rome, I795).

9 A. Boeckhius, Corpus Inscriptionum Graecarum (Berlin, I828-77).

Io 'Euge, euge, le griderò in risposta alla lettera scrittami nel lasciare l'Italia, in cui mi manifesta la risoluzione presa d'ampliare il suo lavoro, ed invece del supplemento alle grandi collezioni epigrafiche da Lei meditato, regalarci l'intero corpo delle iscrizioni latine. Se con tanto plauso è stato ricevuto dall'Europa riconoscente il Corpus inscriptionum Graecarum del cav. Boeckh che pure per la più parte è estraneo affatto, o non risguarda se non che un suo cantone in oggi semibarbaro, con quanto maggiore non accoglierebbe la di Lei fatica tutta appartenente ai suoi antenati e alle sue città? Qual risparmio infinito di sudore e di tempo per gli eruditi, che avrebbero alla mano tutto ciò che la lapidaria può loro offerire, senza dover ripescarlo in un pelago senza fondo, e senza perdere la meta della loro vita in indici, in rimandi, in citazioni.'

I I 'Per quanto riguarda a me, Ella disponga pure della mia cooperazione in tutto ciò che possa crederla di qualche utilità. Oltre la collezione delle figuline, che Le ho già promessa, potrei assolverla di un'altra fatica, ed è quella delle lapidi consolari, comunicandole i miei monumenti ipatici, che mi sarebbero indifferente pubblicare piutosto in un modo, che in un altro. Contengono oltre quattromila iscrizioni disposte cronologicamente sull'esempio della classe quinta del Muratori.'

I2 G. Vagenheim, 'La falsification chez Pirro Ligorio. à la lumière des Fasti Capitolini et des inscriptions de Préneste', Eutopia 3: I-2 (I994), pp. 67-I I3.

I3 Fea publiera également les inscriptions découvertes: Frammenti di fasti consolari e trionfali ultimamente scoperti nel Foro e altrove (Rome, I820).

I4 Dans une lettre du 29 mars de la même année, Borghesi s'était réjoui du succès de Kellermann auprès du gouvernement danois et formulait le vœu que les démarches auprès de l'Académie des sciences de Berlin soient tout aussi fructueuses: 'Mi compiaccio che i passi, i quali vien dando per istabilire le fondamenta della grande impresa di un corpo d'iscrizioni latine, non Le tornino infruttuosi in Danimarca, e Le auguro che altrettanto le accada in Berlino' (p. I49).

I 5 J. Irmschr, 'Die Idee des Umfassenden Inschriftencorpus', Akte des IV. Internationalen Kongresses für Griechische und Lateinische Epigraphik (Wien, I7. bis 22. September 1962) (Graz, I964), p. I67.

I6 Fea, op. cit. (note I3).

I7 Pour plus de détails sur cette polémique, Vagenheim, op. cit. (note I2).

I8 Borghesi venait d'apprendre, par une lettre de Gherard, que le ministre Savigny et l'Académie des sciences de Berlin avaient la ferme intention de diffuser en Allemagne l'étude de l'épigraphie latine (p. 473).

I9 Pour plus de détails sur ce compte rendu, je me permets de renvoyer à G. Vagenheim, 'Le raccolte di iscrizioni di Ciriaco d'Ancona nel carteggio tra Giovanni Battista De Rossi e Theodor Mommsen', dans S. Sconocchia et G. Paci (eds), Ciriaco d'Ancona e la cultura antiquaria dell'umanesimo (Reggio Emilia, I998), pp. 467-5I7.

20 Désormais CIL IX 329: On se consultera aussi M. Chelotti, R. Gaeta, V. Morizio et, M. Silvestrini (eds) Le epigrafe romane di Canosa (Bari, I985).

2 I M. A. Lupuli, Iter Venusinum vetustis monumentis illustratum (Naples, I793), pp. 313, 364. Ida Calabi Limentani avait évoqué cette même inscription dans un article important: 'Bartolomeo Borghesi "disciplinae epigraphicae latinae aetate sua princeps"،, dans Bartolomeo Borghesi, scienza e libertà (Bologna, I982), p. 90. 
22 Sur les rapports entre les deux savants, on consultera avec profit M. Buonocore, Theodor Mommsen e gli studi sul mondo antico: dalle sue lettere conservate nella Biblioteca Apostolica Vaticana (Rome, 2003).

23 G. Vagenheim, 'Les inscriptions ligoriennes. Remarques sur la tradition manuscrite', Italia Medioevale e Umanistica 30 (1987), pp. 199-309, et Silvia Tomasi Velli, Gli antiquari intorno al circo romano: riscoperta di una tipologia monumentale antica (Pisa, I990), pp. 6I-I68.

24 Marco Buonocore, pour sa part, y a identifié la main d'Agustín: 'Onuphrius Panvinius et Antonius Augustinus', dans M. H. Crawford (ed.), Antonio Agustín betmeen Renaissance and Counter-Reform (London, I993), pp. I 55-7I.

25 Voir G. Vagenheim, 'À propos de Valeria Brocchilla (CIL VI 9346). Remarques sur la tradition manuscrite et le classement des inscriptions ligoriennes', Epigraphica 50 (I988), pp. I9I209, et plus récemment A. E. Cooley, The Cambridge Manual of Latin Epigraphy (Cambridge, 2012) et son chapitre sur les fausses inscriptions.
26 G. Kaibel, Inscriptiones Italiae et Siciliae (Berlin, I890). On consultera également S. Orlandi, 'Pirro Ligorio, Mommsen e alcuni documenti epigrafici del Latium adiectum', in F. Mannino, M. Mannino et D. F. Maras (eds), Theodor Mommsen e il Lazio antico (Rome, 2009), pp. 55-62.

27 L. Moretti, 'Inscriptiones graecae ineditae Urbis Romae', Athenaeum 39 (1961), p. 73.

28 IRNL.

29 W. Henzen, ' $\mathrm{Zu}$ den falschungen des Pirro Ligorio', dans Commentationes philologae in honorem Theodori Mommseni (Berlin, I877), pp. 627-43. Voir aussi G. Camodeca et H. Solin, Catalogo delle iscrizioni latine del Museo di Napoli, I. Roma e Latium (Napoli, 2000), qui persistent à considérer certains faux comme des textes authentiques.

30 Cette étude sera désormais facilitée par l'excellente édition des deux volumes épigraphiques les plus importants (xxx.b.7-8) des Antichità romane de Ligorio par Silvia Orlandi, Libri delle iscrizioni latine e greche (Rome, 2008) et Libro delle iscrizioni dei sepolcri antichi (Rome, 2009). 